\title{
Combining genetic and biochemical approaches to identify functional molecular contact points
}

\author{
Matthew P. Badtke ${ }^{1}$, Feng Cao $^{1}$ and John E. Tavis²* \\ ${ }^{1}$ Department of Molecular Microbiology and Immunology, Saint Louis University School of Medicine. St. Louis, MO 63104. USA. \\ ${ }^{2}$ Department of Molecular Microbiology and Immunology and Saint Louis University Liver Center, Saint Louis University School of Medicine. St. Louis, MO \\ 63104. USA. \\ *Corresponding Author: John E. Tavis, Saint Louis University School of Medicine, Department of Molecular Microbiology and Immunology. 1402 S. Grand \\ Blvd., St. Louis, MO 63104. USA. Phone: (314) 977-8893. Fax: (314) 977-8717. Email: tavisje@slu.edu
}

Submitted: May 2, 2006; Revised: July 19, 2006; Accepted: July 19, 2006.

Indexing terms: Hepatitis B Virus, Duck; Protein Interaction Mapping.

\begin{abstract}
Protein-protein interactions are required for many viral and cellular functions and are potential targets for novel therapies. Here we detail a series of genetic and biochemical techniques used in combination to find an essential molecular contact point on the duck hepatitis B virus polymerase. These techniques include differential immunoprecipitation, mutagenesis and peptide competition. The strength of these techniques is their ability to identify contact points on intact proteins or protein complexes employing functional assays. This approach can be used to aid identification of putative binding sites on proteins and protein complexes which are resistant to characterization by other methods.
\end{abstract}

\section{INTRODUCTION}

Protein-protein interactions are essential for most cellular processes. Not surprisingly, many human diseases can be linked to abnormal protein contacts, including some forms of leukemia and neurodegenerative disease (1). Additionally, protein-protein interactions provide therapeutic targets in the treatment of cancer and infectious diseases (2), and development of small molecule inhibitors of protein-protein interactions is a burgeoning field of research.

Several techniques exist to examine protein-protein interactions. Yeast two-hybrid systems [reviewed in (3)] involve fusing proteins to either the DNA binding or transcriptional activation domains of the Gal4 transcription factor. If binding occurs in yeast, a reporter gene is expressed. Glutathione-S-transferase (GST) pulldown is also used to test protein interactions (4). This technique entails expressing GST fusion proteins, allowing them to bind the target protein and purifying the complex by GST-glutathione affinity purification. Similar techniques include co-immunoprecipitation with or without chemical crosslinking. Peptide phage display [reviewed in (5)] involves displaying a library of foreign peptides on the end of coat proteins on the surface of bacteriophage particles. The library is then screened by affinity selection against an immobilized binding partner, and through several rounds peptides with high affinity for the target can be identified. Limitations of these techniques are that they are primarily appropriate for detection of inter-molecular binding and that a positive result indicates these proteins or protein fragments can interact under the conditions of the assay, not that they necessarily do interact under physiological conditions. Specifically, intra-molecular interactions and interactions involving proteins that require numerous cofactors or chaperones to fold properly are difficult or impossible to analyze by these methods. 
One protein that has been extremely difficult to study is the duck hepatitis B virus (DHBV) polymerase (P). DHBV is a common model for the human pathogen hepatitis B virus (HBV). HBV chronically infects more than 350 million people worldwide and is the leading cause of hepatocellular carcinoma (6). These DNA viruses replicate by reverse transcription of an RNA intermediate in which DNA synthesis is primed by $\mathrm{P}$ itself, resulting in covalent attachment of the product DNA to $P$. This feature can be exploited in a priming assay, which measures covalent attachment of radiolabeled dGMP to $P$. Several chaperones are needed for proper folding and function of $\mathrm{P}$, including HSP 90, HSP 70, HSP 40 and Hop $(7,8)$. The requirement of template RNA and cellular chaperone cofactors for $\mathrm{P}$ to become functional has made studying the structure of $\mathrm{P}$ using standard techniques very difficult and has prevented characterization of $\mathrm{P}$ by X-ray crystallography. Therefore, new techniques are needed to understand the folding of $\mathrm{P}$ and to identify inter- or intra-molecular contact sites involving P.

Here we describe a series of biochemical and genetic techniques that we employed to identify an essential molecular contact site on P. Our results and their implications have been published (9). These data will be shown as examples. These approaches can be used to identify putative binding sites within protein complexes resistant to standard approaches. The summary of how we applied this general approach to assess DHBV P is in Table 1.

\begin{tabular}{cll}
\hline $\begin{array}{l}\text { Table } \\
\text { identification. }\end{array}$ & \multicolumn{1}{c}{ Analytical } & strategy for binding site \\
\hline Step & \multicolumn{1}{c}{ General Approach } & \multicolumn{1}{c}{ DHVB P } \\
\hline 1 & $\begin{array}{l}\text { Develop a collection of } \\
\text { sequence-specific } \\
\text { binding agents }\end{array}$ & $\begin{array}{l}\text { Developed a panel of monoclonal } \\
\text { antibodies to the amino-terminal } \\
\text { domain of P }\end{array}$ \\
\hline 2 & $\begin{array}{l}\text { Assess differential } \\
\text { exposure of epitopes } \\
\text { for binding agents in } \\
\text { Step 1 }\end{array}$ & $\begin{array}{l}\text { Immunoprecipitate in low vs. high } \\
\text { detergent buffer }\end{array}$ \\
\hline 3 & $\begin{array}{l}\text { Identify putative } \\
\text { binding sites by } \\
\text { location of epitopes } \\
\text { and sequence analysis }\end{array}$ & $\begin{array}{l}\text { Identified conserved region aa } \\
\text { alignment and position of epitopes } \\
\text { that are differentially exposed }\end{array}$ \\
\hline 4 & $\begin{array}{l}\text { Mutate conserved } \\
\text { residues within } \\
\text { putative binding site }\end{array}$ & $\begin{array}{l}\text { Mutated residues within aa 177- } \\
183\end{array}$ \\
\hline 5 & $\begin{array}{l}\text { Test mutants in } \\
\text { functional assays }\end{array}$ & $\begin{array}{l}\text { Assessed mutants in DNA } \\
\text { priming and partial proteolysis }\end{array}$ \\
\hline 6 & $\begin{array}{l}\text { Compete interaction } \\
\text { with peptides } \\
\text { containing putative } \\
\text { binding site }\end{array}$ & $\begin{array}{l}\text { Dose dependent decrease in } \\
\text { priming when peptide containing } \\
\text { aa 177-183 was added }\end{array}$ \\
\hline
\end{tabular}

\section{MATERIALS AND METHODS}

\section{Materials}

Monoclonal antibodies were generated against $\mathrm{COOH}-$ His tagged terminal protein domain of DHBV P (10). In vitro transcription was performed using the T7 or T3 Megascript kits (Ambion) according to manufacturer's instructions. In vitro translation of $\mathrm{P}$ was performed using rabbit reticulocyte lysate (Promega) with ${ }^{35} \mathrm{~S}$ Methionine (Amersham). Peptides were custom synthesized (Genscript).

\section{Differential immunoprecipitation (Protocol 1)}

Protein A/G beads (Calbiochem) were suspended in either RIPA (20 mM Tris pH 7.2,150 mM NaCl, 1\% Triton $\mathrm{X}-100,1 \%$ sodium deoxycholate, $0.1 \%$ sodium dodecyl sulfate [SDS]) or IPP150 (10 mM Tris $\mathrm{pH} 7.5,150 \mathrm{mM}$ $\mathrm{NaCl}, 0.1 \% \mathrm{NP} 40)$ along with bovine serum albumin $(0.1$ ug/ul). Excess monoclonal antibodies were added to protein $\mathrm{A} / \mathrm{G}$ beads and rocked at $4^{\circ}$. Antibody-bead complexes were washed and then incubated overnight with in vitro translated $\mathrm{P}$ in either RIPA or IPP150. Immunocomplexes were collected and washed with RIPA or IPP150. P was released by boiling in Laemmli Buffer. Samples were resolved by SDS-polyacylamide gel electrophoresis [SDS-PAGE] and exposed to either autoradiography film or a phosphorimager screen.

\section{Bioinformatics}

To find putative binding sites we obtained protein sequences of $\mathrm{P}$ for eight different hepadnaviruses from the National Center for Biotechnology Information (NCBI) Entrez Protein website [http://www.ncbi.nlm.nih.gov/entrez/query.fcgi?db=Prot ein]. The overall sequence identity between these viruses is $26 \%$. We aligned the sequences using Clustal $\mathrm{W}$. Motifs of 4-10 amino acids with $>80 \%$ homology were considered putative binding sites and were investigated further.

\section{DNA priming assay}

$\mathrm{P}$ was translated in vitro in the presence of $\varepsilon$, the RNA required for activation of $\mathrm{P}(11,12)$ and to initiate DNA synthesis $(13,14) .1 \mu \mathrm{l}$ of a $1: 1$ mixture of $48 \mathrm{mM} \mathrm{MgCl}_{2}$ and $\left[\alpha-{ }^{32} \mathrm{P}\right] \mathrm{dGTP}$ was added, and the reaction was incubated at $37^{\circ}$ for 30 minutes. The reaction was 
stopped by addition of Laemmli Buffer, and $\mathrm{P}$ was resolved by SDS-PAGE.

\section{Detection and quantitation of normalized priming signal}

An aliquot of the in vitro translation was removed prior to the priming reaction and resolved by SDS-PAGE to monitor translation efficiency. Because [ $\left.\alpha-{ }^{32} \mathrm{P}\right] \mathrm{dGMP}$ became covalently attached to a ${ }^{35}$ S-labeled protein in the priming reaction, we blocked the ${ }^{35} \mathrm{~S}$ signal by covering the dried gel with a piece of exposed autoradiography film, effectively impeding the ${ }^{35} \mathrm{~S}$ signal while not inhibiting the ${ }^{32} \mathrm{P}$ signal. Both ${ }^{35} \mathrm{~S}$ translation and ${ }^{32} \mathrm{P}$ priming signals from the phosphorimager were analyzed using ImageQuant software (Molecular Dynamics). The priming signal was divided by its cognate translation signal to normalize for translation efficiency.

\section{Partial proteolysis (Protocol 2)}

$\mathrm{P}$ was translated in vitro. Diluted papain (Sigma) was added to a total volume of $20 \mathrm{ul}$ and the mixture was incubated at $14^{\circ}$ for 10 minutes (12). Digestion was stopped by rapidly placing the samples in an ice water slurry followed by addition of 20 ul 2.5X Laemmli sample buffer and boiling for 4 minutes. Digested $\mathrm{P}$ was resolved by SDS-PAGE. Gels were dried and P was detected by phosphorimage analysis.

\section{Monoclonal antibody inhibition of priming}

Monoclonal antibodies were purified by protein A affinity chromatography. These mAbs were added following in vitro translation of $\mathrm{P}$ and allowed to bind to their epitopes. $\varepsilon, \mathrm{MgCl}_{2}$ and $\left[\alpha-{ }^{32} \mathrm{P}\right] \mathrm{dGTP}$ were then added to permit priming (9). The priming signal was normalized to translation efficiency as described above.

\section{Peptide competition (Protocol 3)}

Peptides containing the T3 motif and irrelevant peptides were synthesized and suspended in 100\% dimethyl sulfoxide (DMSO) (Sigma) at $100 \mathrm{mM}$. The peptides were then diluted to $10 \mathrm{mM}$ in $10 \%$ DMSO with water. The final concentration of DMSO in all reactions was $1 \%$. $\mathrm{P}$ was translated in vitro in the presence of $\varepsilon$ and peptide (0.25-1.0 mM), and then $\mathrm{MgCl}_{2}$ and [ $\left.\alpha-32 \mathrm{P}\right] \mathrm{dGTP}$ were added to initiate priming (9). The samples were resolved by SDS-PAGE, the gels were dried and $P$ was detected by phosphorimage analysis. Priming signal was normalized to translation efficiency.

\section{RESULTS}

\section{Identification of systems amenable to this approach}

Conditions under which these approaches would be useful include:

- $\quad$ Protein-protein interactions that are resistant to characterization using standard GST fusion or yeast 2hybrid analyses;

- A source of monoclonal antibodies (mAbs) or similar specific binding agents must be available. If mAbs cannot be made, single chain fragment variable reagents from phage display libraries could be used (15);

- Analytical quantities of functional protein, obtained by purification from bacteria, in vitro translation, baculovirus infection of insect cells or other methods; and

- A functional assay for protein activity.

\section{Differential immunoprecipitation}

Differential immunoprecipitation involves using antibodies or other sequence specific reagents to precipitate a protein under different buffer conditions. We used varying detergent concentrations, high and low, along with six monoclonal antibodies to determine structural changes and the existence of putative contact sites on the DHBV polymerase. In order for this method to be useful, several conditions must be met. First, the antibodies or other sequence-specific binding reagents must recognize one or more motifs on the protein. Also, at least one of the antibodies needs to recognize its epitope and immunoprecipitate (IP) the protein in the high detergent conditions. In our case we used RIPA, a partially denaturing buffer that breaks most weak protein-protein contacts but does not completely denature most proteins. Under less stringent buffer conditions that retain more protein-protein contacts, one or more of the antibodies must now be unable to recognize their target. We chose IPP150 as the low stringency buffer because $\mathrm{P}$ retains sequence-specific RNA binding activity in this buffer (16). If these conditions are met, it can be concluded that detergent treatment can cause conformational changes in the protein and binding sites may be present on the protein. If a binding site is causing the differential immunoprecipitation, occupancy of the binding site in the mild conditions would occlude the antibody epitopes (Fig. 1). Figure 2 shows an example of differential IP using a panel of $\mathrm{mAbs}$ and two different buffers. In the high detergent buffer (RIPA) all of the mAbs recognize their epitopes, while in IPP150 buffer, which has less 
detergent and retains many protein contacts, only mAbs 9, 11 and 12 recognize their epitopes.

A Protein under physiological conditions

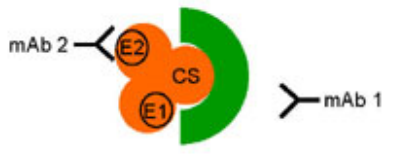

Protein under partially

denaturing conditions
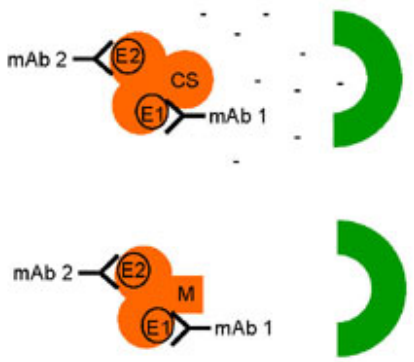

Protein with mutations to the contact site

Protein in the presence of an excess of peptide containing the contact site
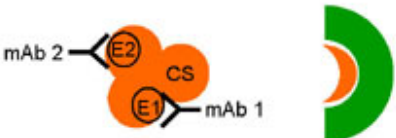

\section{Identification of putative contact sites and mutagenesis}

If differential precipitation has been observed with a sequence-specific reagent, the epitopes the reagent recognizes should be mapped. Once a region of the protein has been implicated by epitope mapping, genetic alignment and mutagenesis can help identify putative contact sites. First, to find potential contact sites, align multiple related protein sequences and look for regions of high homology. An example of a sequence homology alignment using eight different hepadnaviruses is shown in Figure 3. Groups of proteins with very high homology overall will not be amenable to this analysis because there will be too many regions that are similar. Other genetic features that can aid in identification of potential binding sites include hydrophilicity and secondary structure analyses to identify amino acids that may be exposed on the surface of the protein.

$\mathrm{CS}=$ contact site

$E 1=$ monoclonal antibody epitope for mAb that recognizes under select conditions $E 2=$ monoclonal antibody epitope for mAb that recognizes under all conditions

$M=$ mutagenesis at contact site

Fig. 1: Summary of how differential exposure of mAb epitopes can lead to identification of protein-protein contacts. (A) Under normal conditions only some of the antibodies can bind their epitopes due to occlusion of the epitopes by the ligand bound to the contact site. (B) Treatment with partially denaturing buffer dissociates the protein contact site, exposing the monoclonal antibody epitopes. Mutagenesis (C) and peptide competition (D) at the contact site can both disrupt binding of the ligand for the motif and expose the epitopes in a pattern similar to that induced by the partially denaturing buffer.

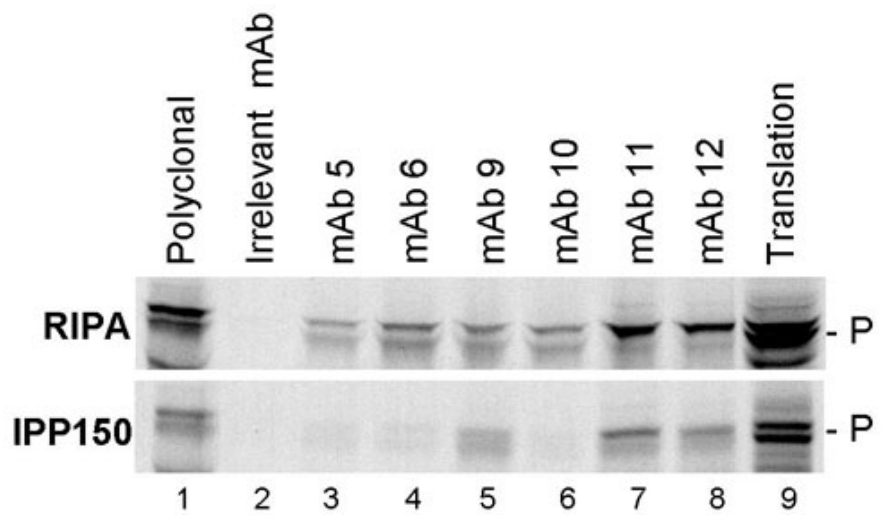

Fig. 2: Differential immunoprecipitation using high and low detergent buffers. An example of differential immunoprecipitation in RIPA and IPP150 buffers. Unprecipitated in vitro translation sample and immunoprecipitation with a polyclonal antibody are controls. Note that mAbs 5, 6 and 10 immunoprecipitate $P$ only under partially denaturing conditions. This figure was originally published in (9).
T3

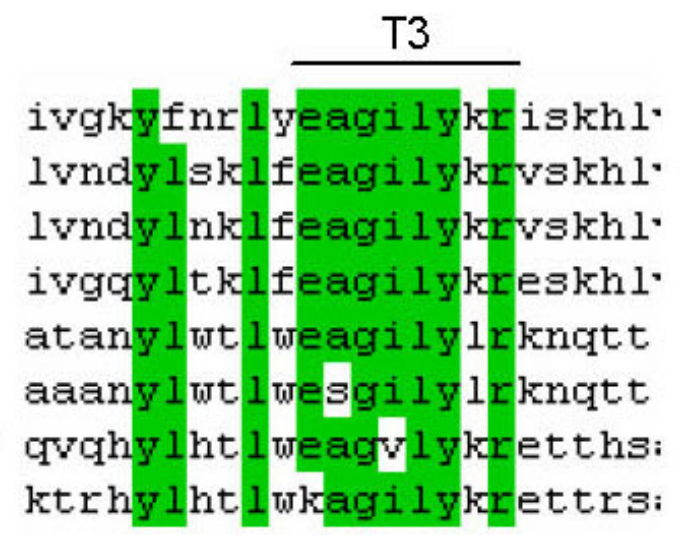

Fig. 3: Sequence homology aligment of multiple hepadnaviruses. $P$ $>80 \%$ homology are shaded green. The T3 motif is indicated. DHBV, Duck hepatitis B virus, SHBV, Stork hepatitis B virus, HHBV, Heron hepatitis B virus, RGHBV, Ross' goose hepatitis B virus, WHV, Woodchuck hepatitis virus, GSHV, Ground squirrel hepatitis virus, WMHBV, Wooly monkey hepatitis B virus, HBV, Hepatitis B virus. Modified from a figure originally published in (9).

Mutagenesis can be used to test if an implicated region is a potential contact site and to determine specific residues involved in binding. If the target residues within the putative contact site are essential for the interaction, their disruption will expose $\mathrm{mAb}$ epitopes normally obscured in low detergent buffer, similar to treatment with partially denaturing buffer (Fig. 1). Figure 4 shows how mutations can expose antibody epitopes that are normally obscured. sequences from eight hepadnaviruses were aligned. Sequences with a 


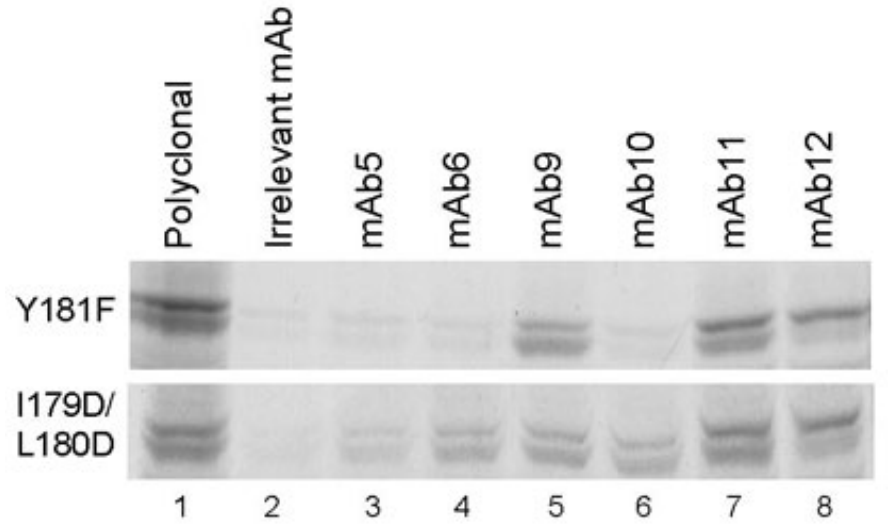

Fig. 4: Mutations can expose epitopes that are normally obscured. $P$ with a mutation that did not affect binding at the contact site (Y181F) or with mutations that did disrupt binding (I179D/L180D) were immunoprecipitated with the indicated antibodies in IPP150 buffer. The epitopes for mAbs 5, 6 and 10 were exposed both by mutations at the contact site and high detergent (compare Figs. 2 and 4). This figure was originally published in (9).

\section{Partial proteolysis}

Partial proteolysis can be used to probe protein structure because proteases recognize and cleave proteins at specific motifs. For example, trypsin cleaves proteins at arginine or lysine residues. The rate of cleavage can be influenced by sequence context and fold of the protein. The initial cleavages on a folded protein will be at surface-exposed residues, leading to a specific partial digestion pattern. If the protein undergoes a conformational change it may alter the exposed residues, causing a change in the partial proteolytic pattern. Therefore, partial proteolysis can be used to test the ability of mutations to alter the structure of a protein. As another measure of effects from our mutants, we assessed their partial proteolysis pattern with papain. Figure 5 illustrates a mutant $P$ with a different protease digestion pattern than wild-type P. Different partial proteolysis patterns show that the mutations affected the structure of the protein, consistent with disrupted binding at the putative contact site.

\section{Monoclonal antibodies as inhibitors of protein activity}

Mabs can be used to probe protein-protein interactions even without differential exposure of their epitopes because binding of an antibody at or near a binding site can sterically inhibit the interaction. Binding can also induce a conformational alteration to the protein and hence affect the function of the protein. The effect of disruption of the binding site can be measured by determining protein function after $\mathrm{mAbs}$ are allowed to bind their epitopes. Mabs that alter the function of the protein must be able to bind their epitopes, whereas those that have no effect on the protein may not be able to bind. This technique provides epitope exposure information and shows direct inhibitory effects of binding. Figure 6 shows the effect that various mAbs have on protein function in DHBV P. Data such as these imply that the epitopes are near important regions and may participate in protein function.

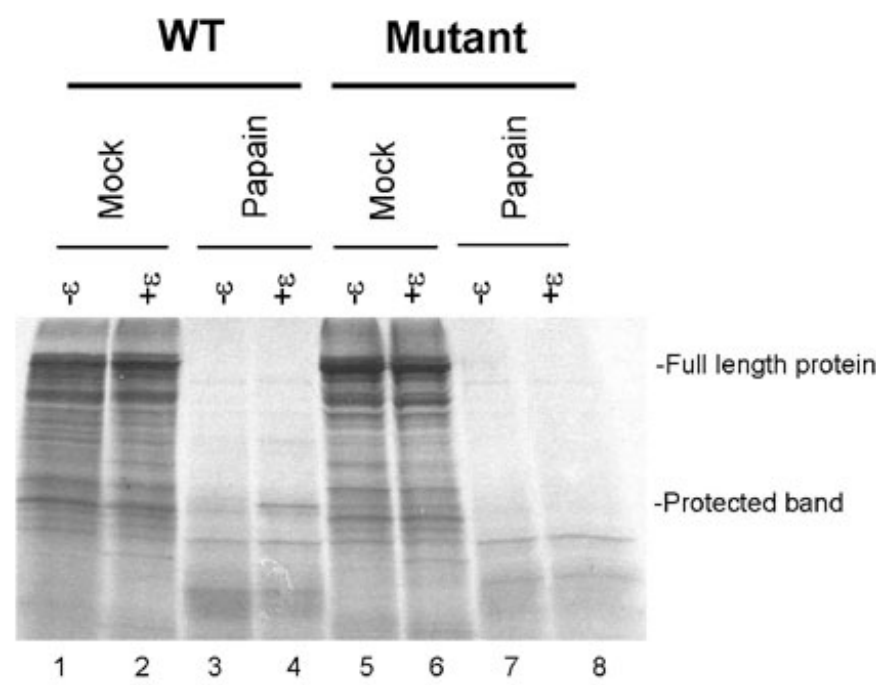

Fig. 5: Mutations can alter partial proteolysis pattern. $P$ was partially digested with papain and the fragments were resolved by SDS-PAGE. Lanes 1-4 show wild-type $P$ and lanes 5-8 show $P$ with mutations which disrupt the contact site on P. $\varepsilon$ is a RNA stem loop required for proper folding and activation of $P$. Note the lack of the protected fragment in the digestion of mutant protein (compare lanes 4 and 8 ).

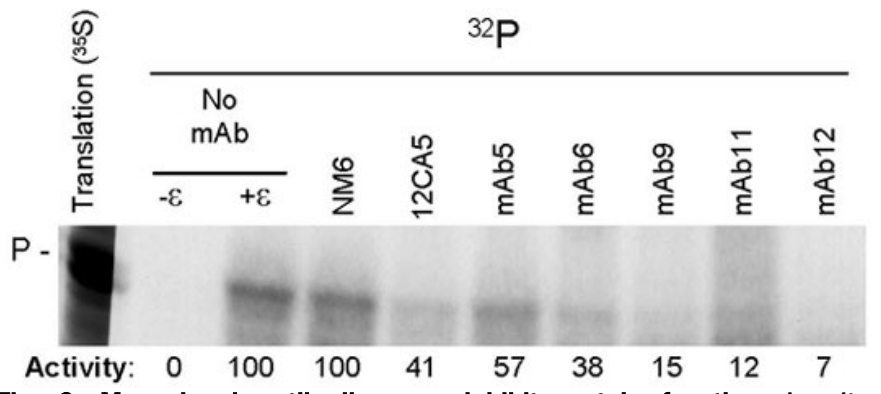

Fig. 6: Monoclonal antibodies can inhibit protein function. In vitro translated $P$ was incubated with the indicated monoclonal antibodies and a DNA priming assay was performed. Priming signals were normalized to activity of P without antibody. NM6 is an irrelevant control antibody. This figure was originally published in (9).

\section{Peptide competition}

Peptide competition can greatly strengthen identification of a protein motif as a binding site. A peptide containing the binding motif can bind its ligand, competitively displacing the normal protein ligand leading to a dose- 
dependent decrease in protein activity. This experiment is useful because the previous techniques only imply a binding interaction. For example, the differential epitope exposure could be due to a change in a conformationally dependent epitope. However, successful displacement of a ligand through peptide competition directly indicates binding. An example of this type of dose-dependent displacement is seen with imidazole competing for binding to $\mathrm{Ni}+$ columns in histidine tag protein purification. Demonstrating specificity through the use of negative control peptides is essential in this assay. An ideal negative control peptide would contain the putative binding site with mutations known to prevent binding. If this is not possible due to solubility issues that can occur with synthetic peptides, a peptide containing the same amino acids whose order has been scrambled is also an excellent control to demonstrate specificity for the putative ligand. Figure 7 is an example of peptide competition using a peptide containing the binding site and an irrelevant peptide. Another interpretation of our results is that the peptide exerts its inhibition by inhibiting folding or binding to a chaperone partner. We have excluded this possibility by showing that a purified active truncation of $\mathrm{P}$, which does not require interactions with any chaperones, is inhibited by the T3 peptide (data not shown).

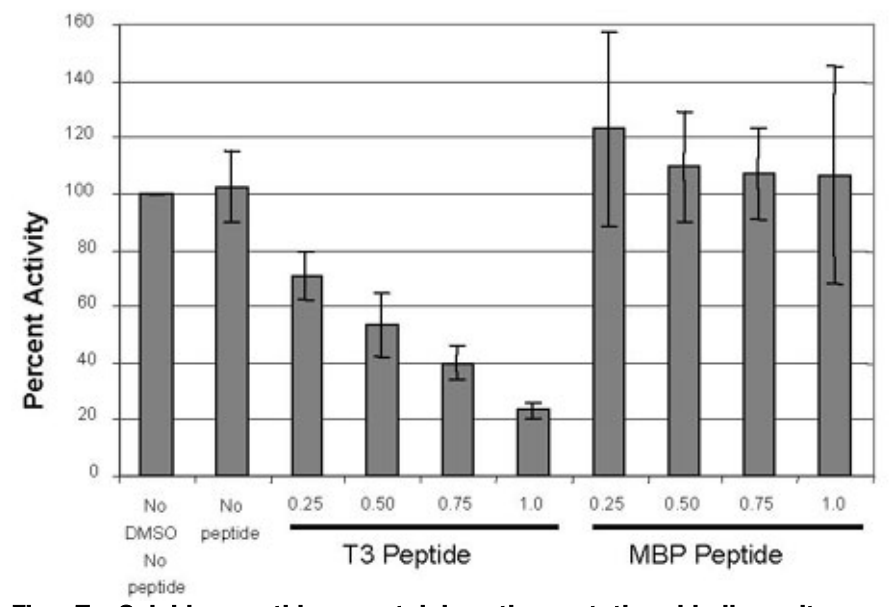

Fig. 7: Soluble peptides containing the putative binding site can competitively inhibit protein function. $P$ was translated in vitro in the presence of the T3 peptide or irrelevant MBP peptide. Priming was initiated by addition of $\mathrm{MgCl}_{2}$ and $\left[\alpha-{ }^{32} \mathrm{P}\right] \mathrm{dGTP}$. Priming signal was normalized to the activity of $\mathrm{P}$ without DMSO or peptide. Peptide concentration is in $\mathrm{mM}$ and error bars are the standard deviation from 4 experiments. This figure was originally published in (9).

\section{DISCUSSION}

Many techniques such as GST-pulldown exist to identify protein-protein interactions, but intra-molecular protein contacts and contacts dependent on the native fold of a protein or formation of a macromolecular complex are difficult if not impossible to analyze by these common techniques. For example, many current techniques detect interactions between protein fragments, which can lead to artifactual positive or false negative results if the fragments do not fold properly. Therefore, there is a need for alternate techniques to address putative proteinprotein interactions. The approach presented here differs from more common techniques as it combines assessment of physical interaction with functional assays using intact proteins under physiological conditions. The strength of the technique is that binding is assessed within unmodified functional proteins or protein complexes. The weakness of this approach is that it cannot formally exclude alternate interpretations of the data such as a role for the identified sites in essential local protein structure. Therefore, whenever possible these approaches should be complimented by data from crosslinking, surface plasmon resonance or similar techniques that measure binding directly.

Protein contact sites are important because they are involved in almost every biological process and are therefore fundamental to cellular function. Furthermore, understanding these contacts provides insight into protein structure, and the contacts themselves can be promising drug targets. Both cellular and viral protein contacts have been targeted for disruption. Small molecules targeting tubulin polymerization (17) and BCL proteins involved in apoptotic signaling [reviewed in (18)] have shown promise as anti-cancer agents. Peptides have been effective in blocking the interaction between the SARS coronavirus and cellular protein angiotensinconverting enzyme 2, the first step in viral infection (19). In DNA viruses, contact sites have been disrupted using both peptides and small molecules, leading to ablation of virus replication. The first example of this was a nonapeptide used to block subunit interaction in the herpes simplex virus ribonucleotide reductase (20). More recently researchers have used peptides to block interactions between subunits of several DNA polymerases [reviewed in $(21,22)]$. This strategy has been successfully demonstrated with viruses such as 
herpes simplex virus (23), human cytomegalovirus (24) and Rous sarcoma virus (25). In addition, peptides have been shown to block interactions in the reverse transcriptase (26), integrase (27) and protease (28) proteins of human immunodeficiency virus. All of these interactions are potential targets for disruption by small molecule inhibitors that could serve as drugs.

\section{ACKNOWLEDGMENTS}

This work was supported by grants AI38447 and CA91327 from the National Institutes of Health. The authors have no conflicts of interest to declare related to this publication.

\section{REFERENCES}

1. Ryan DP, Matthews JM. Protein-protein interactions in human disease. Curr Opin Struct Biol 2005; 15:441446.

2. Arkin M. Protein-protein interactions and cancer: small molecules going in for the kill. Curr Opin Chem Biol 2005; 9:317-324.

3. Miller J, Stagljar I. Using the yeast two-hybrid system to identify interacting proteins. Methods Mol Biol 2004; 261:247-262.

4. Loregian A, Appleton BA, Hogle JM, Coen DM. Specific residues in the connector loop of the human cytomegalovirus DNA polymerase accessory protein UL44 are crucial for interaction with the UL54 catalytic subunit. J Virol 2004; 78:9084-9092.

5. Smith GP, Petrenko VA. Phage display. Chemical Reviews 1997; 97:391-410.

6. Lee WM. Hepatitis B virus infection. N Engl J Med 1997; 337:1733-1745.

7. Hu J, Toft $\mathrm{D}$, Anselmo $\mathrm{D}$, Wang $\mathrm{X}$. In vitro reconstitution of functional hepadnavirus reverse transcriptase with cellular chaperone proteins. J Virol 2002; 76:269-279.

8. Beck J, Nassal M. Efficient Hsp90-independent in vitro activation by $\mathrm{Hsc70}$ and Hsp40 of duck hepatitis B virus reverse transcriptase, an assumed Hsp90 client protein. J Biol Chem 2003; 278:36128-36138.

9. Cao F, Badtke MP, Metzger LM, Yao E, Adeyemo B, Gong $\mathrm{YH}$ et al. Identification of an essential molecular contact point on the duck hepatitis B virus reverse transcriptase. J Virol 2005; 79:10164-10170.
10. Yao E, Gong Y, Chen N, Tavis JE. The majority of duck hepatitis B virus reverse transcriptase in cells is nonencapsidated and is bound to a cytoplasmic structure. J Virol 2000; 74:8648-8657.

11. Tavis JE, Ganem D. Evidence for the Activation of the Hepatitis B Virus Polymerase by Binding of Its RNA Template. J Virol 1996; 70:5741-5750.

12. Tavis JE, Massey B, Gong Y. The Duck Hepatitis B Virus Polymerase Is Activated by Its RNA Packaging Signal, Epsilon. J Virol 1998; 72:5789-5796.

13. Tavis JE, Perri S, Ganem D. Hepadnavirus Reverse Transcription Initiates within the Stem-Loop of the RNA Packaging Signal and Employs a Novel Strand Transfer. J Virol 1994; 68:3536-3543.

14. Wang GH, Seeger C. Novel Mechanism for Reverse Transcription in Hepatitis B Viruses. J Virol 1993; 67:6507-6512.

15. Gibbs RA, Posner BA, Filpula DR, Dodd SW, Finkelman MA, Lee TK et al. Construction and characterization of a single-chain catalytic antibody. Proc Natl Acad Sci USA 1991; 88:4001-4004.

16. Pollack JR, Ganem D. Site-specific RNA binding by a hepatitis $B$ virus reverse transcriptase initiates two distinct reactions: RNA packaging and DNA synthesis. J Virol 1994; 68:5579-5587.

17. Kuo CC, Hsieh HP, Pan WY, Chen CP, Liou JP, Lee SJ et al. BPR0L075, a novel synthetic indole compound with antimitotic activity in human cancer cells, exerts effective antitumoral activity in vivo. Cancer Res 2004; 64:4621-4628.

18. O'Neill J, Manion M, Schwartz P, Hockenbery DM. Promises and challenges of targeting Bcl-2 antiapoptotic proteins for cancer therapy. Biochim Biophys Acta 2004; 1705:43-51.

19. Ho TY, Wu SL, Chen JC, Wei YC, Cheng SE, Chang $\mathrm{YH}$ et al. Design and biological activities of novel inhibitory peptides for SARS-CoV spike protein and angiotensin-converting enzyme 2 interaction. Antiviral Res 2006; 69:70-76.

20. Cohen EA, Gaudreau P, Brazeau P, Langelier Y. Specific inhibition of herpesvirus ribonucleotide reductase by a nonapeptide derived from the carboxy terminus of subunit 2. Nature 1986; 321:441443.

21. Loregian A, Palu G. Disruption of protein-protein interactions: Towards new targets for chemotherapy. J Cellular Physiol 2005; 204:750-762. 
22. Loregian A, Marsden HS, Palu G. Protein-protein interactions as targets for antiviral chemotherapy. Reviews in Medical Virol 2002; 12:239-262.

23. Loregian A, Palu G. Disruption of the interactions between the subunits of herpesvirus DNA polymerases as a novel antiviral strategy. Clin Microbiol Infection 2005;11:437-446.

24. Loregian A, Rigatti R, Murphy M, Schievano E, Palu G, Marsden HS. Inhibition of human cytomegalovirus DNA polymerase by C-terminal peptides from the UL54 subunit. J Virol 2003; 77:83368344.

25. Murray J, Loney C, Murphy LB, Graham S, Yeo RP. Characterization of monoclonal antibodies raised against recombinant respiratory syncytial virus nucleocapsid $(\mathrm{N})$ protein: Identification of a region in the carboxy terminus of $\mathrm{N}$ involved in the interaction with P protein. Virology 2001; 289:252-261.

26. Morris MC, Robert-Hebmann V, Chaloin L, Mery J, Heitz F, Devaux C et al. A new potent HIV-1 reverse transcriptase inhibitor - A synthetic peptide derived from the interface subunit domains. J Biol Chem 1999; 274:24941-24946.

27. Maroun RG, Krebs D, Roshani M, Porumb H, Auclair C, Troalen F et al. Conformational aspects of HIV-1 integrase inhibition by a peptide derived from the enzyme central domain and by antibodies raised against this peptide. Eur J Biochem 1999; 260:145-155.

28. Zhang ZY, Poorman RA, Maggiora LL, Heinrikson RL, Kezdy FJ. Dissociative inhibition of dimeric enzymes. Kinetic characterization of the inhibition of HIV-1 protease by its $\mathrm{COOH}$-terminal tetrapeptide. J Biol Chem 1991; 266:15591-15594. 


\section{PROTOCOLS}

\section{Protocol 1: Differential immunoprecipitation}

1. Prepare solid phase: Suspend $20 \mathrm{ul}$ of protein A/G suspension per sample in $1 \mathrm{ml}$ RIPA or IPP150 buffer. Pellet agarose, aspirate medium and replace with $300 \mathrm{ul}$ buffer containing $0.1 \mathrm{ug} / \mathrm{ul}$ bovine serum albumin.

2. Bind antibody to solid phase: Add antibody to the protein A/G agarose suspension. The amount needs to be determined for each antibody used. Often 1-5 ul of a polyclonal antibody or 1-50 ul of a monoclonal antibody is sufficient. Rock at $4^{\circ}$ for 1-2 hours. Wash beads twice with $1 \mathrm{ml}$ cold buffer and suspend protein A/G agarose in 50 ul RIPA or IPP150 buffer per sample to be immunoprecipitated.

3. Bind antibody to target protein: Add $10 \mathrm{ul}$ of protein to $190 \mathrm{ul}$ of RIPA or IPP150 buffer. Add $50 \mathrm{ul}$ of the appropriate antibody/protein A/G agarose. Rock at $4^{\circ}$ for 1.5 hours to overnight.

4. Wash immunocomplexes: Centrifuge for 30 seconds in a microcentrifuge. Aspirate the supernatant and add $1 \mathrm{ml}$ cold RIPA or IPP150 buffer and vortex for 30 seconds. Repeat 3 times. Centrifuge samples for 1 minute and remove all remaining liquid, keeping the pellet intact.

5. Prepare samples for electrophoresis: Add $25 \mathrm{ul}$ of Laemmli sample buffer to the pellet. Vortex to resuspend the pellet and boil for 4 minutes. Cool the sample on ice and centrifuge 2 minutes. Transfer liquid to new tube, leaving the pellet behind.

6. Electrophoresis: Resolve 5-20 ul of the sample on an appropriate percentage polyacrylamide gel.

7. Analysis of sample: Detect protein by autoradiography (if radioactive) or Western blot. Be sure to include a sample of un-immunoprecipitated protein as an immunoprecipitation yield control.

\section{Solutions needed}

IPP150 - 10 mM Tris pH 7.5, $150 \mathrm{mM} \mathrm{NaCl,} \mathrm{0.1 \%} \mathrm{NP40}$

RIPA - 20 mM Tris pH 7.2, $150 \mathrm{mM} \mathrm{NaCl}$, 1\% Triton X-100, 1\% sodium deoxycholate, $0.1 \%$ sodium dodecyl sulfate

Laemmli protein sample buffer (5X) - 10\% sodium dodecyl sulfate, 20\% glycerol, 125mM Tris $\mathrm{pH} 6.8,0.1 \%$ Bromophenol blue, 10\% 2-mercaptoethanol

\section{Protocol 2: Partial protease digestion}

1. Prepare protein.

2. Dilute protease into ice-cold water. Exact dilution needs to be determined for each stock of enzyme.

3. Transfer $5 \mathrm{ul}$ of protein to a fresh pre-chilled tube on ice.

4. Add 5 ul water to one set of tubes and place on ice (mock digestion).

5. Add 5 ul diluted protease to other set of tubes, making sure they stay ice cold.

6. Place all tubes at $14^{\circ}$ for 10 minutes. Rapidly terminate digestion by placing entire set of tubes directly into ice water when digestion is finished. Adjust time, temperature, concentration of protease, and type of protease used empirically until reproducible partial proteolysis is achieved.

7. Stop the reaction by adding $20 \mathrm{ul} 2 \mathrm{X}$ Laemmli sample buffer on ice.

8. Boil samples for 4 minutes and load 10-15 ul on a polyacrylamide gel.

9. Analyze samples as described in Protocol 1.

\section{Solutions needed}

Laemmli protein sample buffer (5X) - See Protocol 1. 


\section{Protocol 3: Peptide competition assay}

1. Dissolve peptides in dimethyl sulfoxide (DMSO), and then dilute with water to a final concentration of $10 \mathrm{mM}$ and $10 \%$ DMSO.

2. Add peptides to the in vitro translation mix:

- 14 ul rabbit reticulocyte lysate

- $\quad 0.4$ ul RNAsin (Promega)

- $\quad 0.4$ ul AA mix (minus methionine)

- 1.2 ul [35S]Methionine

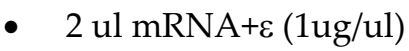

- 2 ul DEPC water, $10 \%$ DMSO or peptide

- 20 ul total volume

Incubate at $30^{\circ}$ for 60 minutes. Stop translation by adding cyclohexamide (final concentration $80 \mu \mathrm{M}$ ). Include translation controls.

3. Add $2 \mu$ of $1: 1$ mixture of $48 \mathrm{mM} \mathrm{MgCl}_{2}:[\alpha-32 \mathrm{P}] \mathrm{dGTP}(10 \mathrm{mCi} / \mathrm{ml})$ to permit DNA priming.

4. Stop reaction by adding $30 \mathrm{ul}$ of $2 \mathrm{X}$ Laemmli protein sample buffer.

5. Boil samples for 4 minutes and load $15 \mathrm{ul}$ on a polyacrylamide gel.

6. Fix, dry and expose gels to autoradiography film or phosphorimager with a sheet of exposed film covering the priming samples to block ${ }^{35} \mathrm{~S}$ signals.

7. Quantitate ${ }^{35} \mathrm{~S}$ and ${ }^{32} \mathrm{P}$ signals by densitometry or phoshorimage analysis. Normalize signal to P without DMSO or peptide.

\section{Solutions needed}

Laemmli protein sample buffer (5X) - See Protocol 1. 